\title{
Desarrollo de un indicador para el análisis de sustentabilidad en obras de hormigón, en Argentina
}

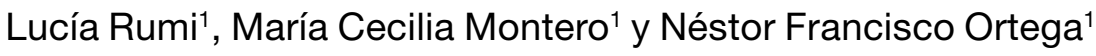

\section{Resumen}

El hormigón es uno de los materiales de mayor consumo mundial debido a su gran cantidad de aplicaciones y durabilidad. También es uno de los que mayores afectaciones genera al ambiente. Por estos motivos, y considerando que en un futuro cercano será difícil que se pueda reemplazar al hormigón, resulta imprescindible que se estudie, la forma de prevenir y reducir los impactos ambientales negativos que generan estas construcciones. En este trabajo se presenta una metodología para establecer criterios de evaluación de la sustentabilidad de las construcciones y, en particular, se desarrollan los indicadores que componen el criterio ambiental. La aplicación los mismos permite cuantificar la sustentabilidad de una construcción de hormigón, con una metodología relativamente simple. Se espera que el uso de este tipo de indicadores posibilite la adopción de estrategias de gestión sustentables, de quienes tienen en su poder la toma de decisiones que mejoren o mantengan las condiciones de sustentabilidad en el ámbito de la construcción.

Palabras clave: Indicadores; sustentabilidad; hormigón; construcciones. 


\begin{abstract}
Concrete is one of the most consumed materials around the world because of its many applications and durability. It is also one of the materials that generates the greatest damage to the environment. For these reasons, and considering that in the future it will be difficult to replace the concrete, it is essential to study the way to prevent and reduce the negative environmental impacts generated by these constructions. This paper presents a methodology to establish the criteria for assessing the sustainability of buildings, and in particular to develop the indicators involved in the environmental criteria. The application of these indicators allows the quantification of the sustainability of a concrete construction, with a relatively simple methodology. It is expected that the use of this type of indicator will help to improve the adoption of sustainable management strategies of those who are holding the decision to improve or maintain the conditions of sustainability in the construction field.
\end{abstract}

Key words: Indicators; sustainability; concrete; constructions. 


\section{Introducción}

Haciendo foco en la actividad de la construcción civil, este sector posee una importancia significativa en el logro del desarrollo sostenible. Las actividades de construcción, uso, reparación, mantenimiento y demolición consumen recursos y generan residuos (ALCONPAT Brasil, 2013). Según informe del Programa de las Naciones Unidas para el Medio Ambiente y la SBCI (Sustainable Building and Construction Iniciative) la industria de la construcción consume entre el $25 \%$ y el $40 \%$ del consumo global de energía, genera entre el $30 \%$ y $40 \%$ de los residuos sólidos y el 20\% de los residuos líquidos y emite entre el 30\% y $40 \%$ de los gases efecto invernadero (ALCONPAT Brasil, 2013).

Considerando que el hormigón es uno de los materiales de mayor consumo mundial debido a su gran cantidad de aplicaciones y durabilidad, es de destacar el rol de la industria del cemento. Esta industria posee desafíos importantes, en cuanto a los cada vez mayores costos de la energía, la necesidad de disminuir las emisiones y mantener el abastecimiento de la materia prima en calidad y cantidad (ALCONPAT Brasil, 2013). Por otro lado, la producción mundial anual de cemento ocasiona aproximadamente el $7 \%$ de la carga total de dióxido de carbono en la atmósfera de origen antropogénico. Según el World Business Council for Sustainable Development la industria del cemento ha alcanzado en los últimos años un desacople importante entre el crecimiento del volumen de producción y las emisiones de CO2 (Schneider, et. al, 2011).

Como ya se indicó anteriormente, el hormigón es uno de los materiales de construcción más utilizados en el mundo, esto se ve agravado por el hecho que, la producción de cemento portland, un componente esencial de hormigón, conduce a la liberación de gran cantidad de $\mathrm{CO} 2$, un gas de efecto invernadero (Naik, et. al, 2005). La producción de 1 tonelada de clinker de cemento portland requiere aproximadamente 4 GJ de energía, y su fabricación libera aproximadamente 1 tonelada de dióxido de carbono y otros gases de efecto invernadero (GEI) a la atmósfera. Las grandes cantidades de extracción de materias primas tales como caliza y arcilla, y el combustible como el carbón, a menudo resultan en una deforestación extensiva y pérdida de suelo superficial (Metha, 2001).

Por estos motivos, las cuestiones ambientales tendrán un papel destacado en el desarrollo sustentable de la industria del cemento y el hormigón (Naik, et. al. 2005).

El hormigón es el material más ampliamente utilizado con fines estructurales. Entre sus ventajas, se pueden enunciar: versatilidad (pocas limitaciones geométricas), buen comportamiento en servicio, material económico, etc.

Por estos motivos, y considerando que en un futuro cercano será difícil que se pueda reemplazar al hormigón, resulta imprescindible que se estudie, la forma de prevenir y reducir los impactos ambientales negativos que generan estas 
construcciones (Montero, et. al, 2013).

En relación a los materiales de uso en la construcción civil, los minerales no metálicos responden por el $40 \%$ de la extracción total de materiales en el mundo. En el año 2002 se extrajeron 22.9 billones de toneladas de minerales no metálicos. Este tipo de minerales posee relativo bajo impacto ambiental por tonelada, en comparación con los minerales metálicos y/o fuentes fósiles, pero debido a los volúmenes de materiales que involucra, posee implicancias ambientales significativas, como alteraciones en paisajes, destrucción de hábitat, ruido, cambios en los cursos de ríos, uso intenso de energía, entre otros (ALCONPAT Brasil, 2013).

\section{Criterios - indicadores}

La sustentabilidad es un concepto global no restringido al ámbito de la construcción, que requiere que se satisfagan una serie de criterios ambientales, así como otros de carácter económico y social. En este trabajo se desarrollará el criterio ambiental. En tal sentido, se propone un conjunto de indicadores destinados a evaluar, en forma integral, las condiciones de sustentabilidad de una construcción portuaria, de hormigón, el que dependerá del cumplimiento de ciertos criterios, varios de ellos basados en lo establecido por la Instrucción Española de Hormigón Estructural (EHE, 2008).

Para su elaboración fue necesario, en primer término, la caracterización sistémica del espacio bajo análisis. Esta tarea facilitó la posterior identificación de las variables centrales de análisis, que sirvieron de línea de base para la formulación de los indicadores destinados a evaluar y monitorear la sustentabilidad. Se espera que la concreción de estos objetivos posibilite la adopción de estrategias de gestión sustentables, de quienes tienen en su poder la toma de decisiones que mejoren o mantengan las condiciones de sustentabilidad en el ámbito de la construcción.

Es importante comentar que los indicadores desarrollados en este trabajo son aplicables a obras de ingeniería civil en las que la construcción de hormigón es predominante, con respecto al resto de la obra, tal es el caso de puertos, puentes y presas de hormigón. Para un análisis integral de cualquiera de estos casos, a los indicadores que se presentan en este trabajo habría que añadirle el análisis de rubros importantes de la obra, como ser movimiento de suelos. Una presa, habitualmente, requiere voladuras de rocas y movimientos importantes de suelos, mientras que en un puerto es muy frecuente que se requiera realizar tareas de dragado, siendo muy importante el análisis ambiental de estos ítems, además del lugar de depósito del material extraído. Como se puede suponer, los análisis ambientales de este rubro en el caso de la presa y en el puerto son muy distintos, por ello, sería conveniente clasificar cada tipología de obra para poder así estudiarlas por separado. Por otra parte, también se debería analizar en cada caso particular el impacto global de la construcción sobre el lugar de emplazamiento, 
teniendo en cuenta criterios ecológicos, geomorfológicos, impactos visuales, entre otros. Estos factores escapan al objetivo del presente trabajo, que se circunscribe a las cuestiones estrictamente relacionadas a la estructura de hormigón. Asimismo, el estudio ambiental de algunos de estos factores, podrían originar publicaciones de similar o mayor complejidad que la presente. En particular, el caso de obras portuarias, con las consideraciones antes mencionadas, está siendo estudiado por uno de los autores de este trabajo, formando parte de su tesis de maestría.

\section{Metodología}

La metodología propuesta se desarrolla con el fin de evaluar los impactos producidos durante la construcción de la obra, subdividiéndolo en dos vías de estudio:

- Impactos de los materiales y productos básicos en la elaboración del hormigón.

Como resultado de este análisis obtendremos un Índice de los Productos Básicos (IPB).

- Impactos adicionales, que tienen que ver con el proceso constructivo. Como resultado obtendremos un Índice del Proceso Constructivo (IPC).

Finalmente se ensamblan estas dos vías obteniendo un solo índice, llamado Índice de Sustentabilidad del Hormigón (ISH):

$$
\begin{gathered}
I S H=0,5 \cdot I P B+0,5 \cdot I P C \\
0 \leq I S H \leq \mathbf{1}
\end{gathered}
$$

\subsection{Definición del Índice de Productos Básicos}

El IPB se basa en el índice ISMA, correspondiente al método de la Instrucción de Hormigón Estructural (EHE, 2008). Para ello, se tuvo en cuenta el contexto local, tanto las normativas de calidad (ISO 14001, 2004) como los medios y distancias de transporte, en este trabajo se consideró que es uno de los aspectos de mayor importancia para la ponderación de los indicadores, a diferencia de lo que marca la normativa española.

Se define como "Índice de Productos Básicos" (IPB) de una construcción al resultado de aplicar la siguiente expresión:

donde:

$$
I P B=\sum_{i=1}^{n} C C i \cdot V i
$$

- $\mathrm{CC}_{i}$ : Coeficiente compuesto

- $\mathrm{V}_{i}$ : Coeficientes de valor obtenidos para cada criterio. 


\subsubsection{Coeficiente Compuesto $\mathrm{CC}_{i}$}

Se define un coeficiente compuesto para ponderar distintos aspectos, relacionados con los materiales y las medidas para reducir los impactos, de la siguiente manera:

donde:

$$
C C i=\alpha i \cdot \beta i \cdot \gamma i
$$

- $\alpha i, \beta i$ y $\gamma i$ : Coeficientes de ponderación de cada criterio. Se adoptaron los mismos coeficientes que los correspondientes a la norma española (Tabla 1) (EHE 2008).

Tabla 1: Coeficientes de ponderación del $I P B$.

\begin{tabular}{|c|c|c|c|c|c|c|}
\hline \multicolumn{7}{|c|}{ COEFICIENTES } \\
\hline \multicolumn{2}{|l|}{$a_{i}$} & \multicolumn{2}{|l|}{$\boldsymbol{\beta}_{i}$} & \multicolumn{2}{|l|}{$\gamma_{i}$} & $C C i$ \\
\hline \multirow{4}{*}{ Productos } & \multirow{4}{*}{0,60} & \multirow{2}{*}{$\begin{array}{l}\text { Tipo y optimización } \\
\text { de los productos } \\
\text { básicos }\end{array}$} & \multirow{2}{*}{0,55} & $\begin{array}{c}\text { Caracterización del } \\
\text { hormigón }\end{array}$ & 0,50 & 0,165 \\
\hline & & & & $\begin{array}{c}\text { Caracterización del } \\
\text { acero }\end{array}$ & 0,50 & 0,165 \\
\hline & & \multirow{2}{*}{$\begin{array}{l}\text { Preservación de } \\
\text { recursos }\end{array}$} & \multirow{2}{*}{0,45} & $\begin{array}{l}\text { Empleo de áridos } \\
\text { reciclados }\end{array}$ & 0,40 & 0,108 \\
\hline & & & & $\begin{array}{c}\text { Optimización del } \\
\text { cemento }\end{array}$ & 0,60 & 0,162 \\
\hline \multirow{3}{*}{$\begin{array}{c}\text { Medidas } \\
\text { para reducir } \\
\text { los } \\
\text { impactos }\end{array}$} & \multirow{3}{*}{0,40} & $\begin{array}{c}\text { Impacto en el entorno } \\
\text { humano }\end{array}$ & 0,25 & Control de impactos & 1,00 & 0,100 \\
\hline & & \multirow{2}{*}{$\begin{array}{l}\text { Impacto en el } \\
\text { ambiente }\end{array}$} & \multirow{2}{*}{0,75} & Gestión de residuos & 0,67 & 0,201 \\
\hline & & & & Gestión del agua & 0,33 & 0,099 \\
\hline
\end{tabular}

\subsubsection{Determinación de los valores Vi}

En el caso de los materiales y productos básicos para la construcción de la obra, este valor se obtiene a partir de los datos del tipo de material, su cantidad y calidad, distancia y tipo de transporte utilizado, multiplicando la puntuación correspondiente a cada ítem (Tabla 2). 
Tabla 2: Determinación de los valores $V$ i para el $I P B$.

\begin{tabular}{|c|c|c|c|c|}
\hline \multirow{2}{*}{\multicolumn{2}{|c|}{\begin{tabular}{|c|}
$\begin{array}{c}\text { Caracterización del } \\
\text { hormigón }\end{array}$ \\
Calidad: Cumple ISO 14000 \\
\end{tabular}}} & \multicolumn{3}{|c|}{$V_{1}=P_{11} \cdot P_{12} \cdot P_{13} \cdot P_{14}$} \\
\hline & & $\mathrm{Si} \rightarrow P_{11}=1$ & No $\rightarrow$ & $P_{11}=0,7$ \\
\hline \multirow{2}{*}{\multicolumn{2}{|c|}{ Tipo de hormigón }} & Prefabricado & \multirow{2}{*}{$\begin{array}{c}\begin{array}{c}\text { Elaborado en } \\
\text { planta externa }\end{array} \\
P_{12}=0,7 \\
\end{array}$} & $\begin{array}{c}\text { Elaborado } \\
\text { en planta, } \\
\text { en obra }\end{array}$ \\
\hline & & $P_{12}=1$ & & $P_{12}=0,5$ \\
\hline \multirow{4}{*}{ Transporte } & Distancia & $\begin{array}{l}P_{13}=1,9846 \mathrm{x}^{-0,67} \\
\mathrm{X:} \text { distancia de } \\
\text { transporte }[\mathrm{Km}]\end{array}$ & $\begin{array}{c}P_{13}=3,158 x^{-0,67} \\
X: \text { distancia de } \\
\text { transporte }[\mathrm{Km}]\end{array}$ & $P_{13}=1$ \\
\hline & \multirow{3}{*}{ Tipo } & Tren $\rightarrow P_{14}=1$ & \multirow{3}{*}{$P_{14}=1$} & \multirow{3}{*}{$P_{14}=1$} \\
\hline & & Camión $\rightarrow P_{14}=0,7$ & & \\
\hline & & $\begin{aligned} \text { Para dist. } & <100 \mathrm{Km} \\
P_{14} & =1\end{aligned}$ & & \\
\hline \multicolumn{2}{|c|}{ Caracterización del acero } & \multicolumn{3}{|c|}{$V_{2}=P_{21} \cdot P_{22} \cdot P_{23}$} \\
\hline & $\mathrm{Si} \rightarrow P_{21}=1$ & \multicolumn{2}{|c|}{ No $\rightarrow P_{21}=0,7$} \\
\hline Calidad: Cumple ISO 14000 & Distancia & \multicolumn{3}{|c|}{$\begin{array}{c}P_{22}=19,39 \mathrm{x}^{-0,67} \\
\mathrm{x}: \text { distancia de transporte }[\mathrm{Km}]\end{array}$} \\
\hline \multirow[t]{2}{*}{ Transporte } & \multirow{2}{*}{ Tipo } & Tren $\rightarrow P_{23}=1$ & \multicolumn{2}{|c|}{ Camión $\rightarrow P_{23}=0,7$} \\
\hline & & \multicolumn{3}{|c|}{ Para distancias menores a $100 \mathrm{Km}, P_{23}=1$} \\
\hline \multicolumn{2}{|c|}{$\begin{array}{l}\text { Empleo de áridos } \\
\text { reciclados }\end{array}$} & \multicolumn{3}{|c|}{$V_{3}=P_{31} \cdot P_{32} \cdot P_{33} \cdot P_{34}$} \\
\hline \multicolumn{2}{|c|}{$\begin{array}{l}\text { Cantidad: Se permite hasta } \\
\text { un } 25 \% \text { de agregados } \\
\text { reciclados. }\end{array}$} & \multicolumn{3}{|c|}{$\begin{array}{l}\text { Aridos reciclados/Totales } \leq 25 \% \rightarrow P_{31}=0,04 \mathrm{x} \\
\quad \mathrm{x} \text { : Aridos reciclados/Totales, en peso }\end{array}$} \\
\hline \multicolumn{2}{|c|}{$\begin{array}{l}\text { Calidad: Cumple con } \\
\text { CIRSOC 201, aplicado a un } \\
\text { Hormigón con agregados } \\
\text { reciclados. }\end{array}$} & $\mathrm{Si} \rightarrow P_{32}=1$ & \multicolumn{2}{|c|}{ No $\rightarrow P_{32}=0,7$} \\
\hline \multirow{3}{*}{ Transporte } & Distancia & \multicolumn{3}{|c|}{$\begin{array}{c}P_{33}=5,8357 \mathrm{x}^{-0,67} \\
\mathrm{x}: \text { distancia de transporte }[\mathrm{Km}]\end{array}$} \\
\hline & \multirow{2}{*}{ Tipo } & Tren $\rightarrow P_{34}=1$ & \multicolumn{2}{|c|}{ Camión $\rightarrow P_{34}=0,7$} \\
\hline & & \multicolumn{3}{|c|}{ Para distancias menores a $100 \mathrm{Km}, P_{34}=1$} \\
\hline \multicolumn{2}{|c|}{ Optimización del cemento } & $V_{4}=P_{41}$ & $P_{42} \cdot P_{43} \cdot P_{44} \cdot P_{45} \cdot$ & \\
\hline $\begin{array}{l}\text { Cantidad: } \\
x: \text { cantidad c } \\
\text { peso, por } \mathrm{m}^{3} \\
{\left[\mathrm{Kg} / \mathrm{m}^{3}\right]} \\
\end{array}$ & $\begin{array}{l}\text { o, en } \\
\text { rmigón }\end{array}$ & $\begin{array}{r}x \leq 380 \mathrm{Kg} / \mathrm{m} \\
x>380 \mathrm{Kg} / \mathrm{m}^{3}\end{array}$ & $\begin{array}{l}\rightarrow P_{41}=0,0235 \mathrm{x} \\
\rightarrow P_{41}=-0,0143 \mathrm{x} \\
0 \leq P_{41} \leq 1\end{array}$ & $\begin{array}{l}7059 \\
, 2857\end{array}$ \\
\hline Calidad: Cur & ISO 14000 & $\mathrm{Si} \rightarrow P_{42}=1$ & No $\rightarrow$ & $P_{42}=0,7$ \\
\hline & Distancia & $\begin{array}{r}P_{4} \\
\mathrm{x}: \text { distan } \\
\end{array}$ & $\begin{array}{l}=19,39 x^{-0,67} \\
\text { de transporte }[\mathrm{K}\end{array}$ & \\
\hline Transporte & Tino & Tren $\rightarrow P_{44}=$ & Camión & $P_{44}=0,7$ \\
\hline & & Para distancias & menores a $100 \mathrm{Km}$ & $P_{44}=1$ \\
\hline
\end{tabular}

También se evalúa la aplicación o no de determinadas medidas para reducir los impactos, a través de una puntuación (Tabla 3 ). 
Tabla 3: Valores de puntuación para reducir los impactos del $I P B$.

\begin{tabular}{|l|c|c|}
\hline \multirow{2}{*}{\multicolumn{1}{|c|}{ Sub-ítem }} & \multicolumn{2}{c|}{ Puntuación de respuestas } \\
\cline { 2 - 3 } & Positivas & Negativas \\
\hline Control de impactos & 0,2 & 0 \\
\hline Gestión de residuos & 1 & 0 \\
\hline Gestión del agua & 0,5 & 0 \\
\hline
\end{tabular}

La sumatoria de las puntuaciones será multiplicada por el coeficiente de ponderación correspondiente a cada sub-ítem, tal como se indica en la Tabla 4.

\subsubsection{Cálculo del IPB}

Resumen del método de obtención del índice IPB (Tablas 4 y 5).

Tabla 4: Determinación del $I P B$.

\begin{tabular}{|c|c|c|c|c|c|}
\hline \multicolumn{6}{|c|}{ PRODUCTOS } \\
\hline \multirow{9}{*}{ 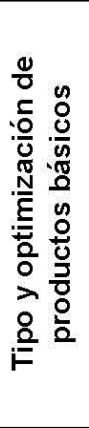 } & \multicolumn{3}{|c|}{ Caracterización del Hormigón } & $C C_{1}$ & $V_{1}$ \\
\hline & \multicolumn{2}{|l|}{ Calidad } & $P_{11}=$ & \multirow{4}{*}{0,165} & \\
\hline & \multicolumn{2}{|l|}{ Tipo } & $P_{12}=$ & & \\
\hline & \multirow{2}{*}{ Transporte } & Distancia & $P_{13}=$ & & \\
\hline & & Tipo & $P_{14}=$ & & \\
\hline & \multicolumn{3}{|c|}{ Caracterización del la armaduras } & $C C_{2}$ & $V_{2}$ \\
\hline & \multicolumn{2}{|l|}{ Calidad } & $P_{21}=$ & \multirow{3}{*}{0,165} & \\
\hline & \multirow{2}{*}{ Transporte } & Distancia & $P_{22}=$ & & \\
\hline & & Tipo & $P_{23}=$ & & \\
\hline \multirow{10}{*}{ 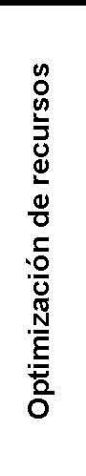 } & \multicolumn{3}{|c|}{ Reciclado de áridos } & $C C_{3}$ & $V_{3}$ \\
\hline & \multicolumn{2}{|l|}{ Cantidad } & $P_{31}=$ & \multirow{4}{*}{0,108} & \\
\hline & \multicolumn{2}{|l|}{ Calidad } & $P_{32}=$ & & \\
\hline & \multirow{2}{*}{ Transporte } & Distancia & $P_{33}=$ & & \\
\hline & & Tipo & $P_{34}=$ & & \\
\hline & \multicolumn{3}{|c|}{ Optimización del Cemento } & $\mathrm{CC}_{4}$ & $V_{4}$ \\
\hline & \multicolumn{2}{|l|}{ Cantidad } & $P_{41}=$ & \multirow{4}{*}{0,162} & \\
\hline & \multicolumn{2}{|l|}{ Calidad } & $P_{42}=$ & & \\
\hline & \multirow{2}{*}{ Transporte } & Distancia & $P_{43}=$ & & \\
\hline & & Tipo & $P_{44}=$ & & \\
\hline
\end{tabular}


Tabla 5: Determinación del $I P B$.

\begin{tabular}{|c|c|c|c|c|}
\hline \multicolumn{5}{|c|}{ MEDIDAS PARA REDUCIR LOS IMPACTOS } \\
\hline Control de Impactos & SI & NO & $C C_{s}$ & $V_{s}$ \\
\hline Empleo de aspersores en la obra para evitar el polvo & & & & \\
\hline $\begin{array}{l}\text { Pavimentación de los accesos a la obra o inclusuión de } \\
\text { sistemas de limpieza de neumáticos }\end{array}$ & & & & \\
\hline $\begin{array}{l}\text { Ulitización de pantallas u otros dispositivos de retención } \\
\text { de polvo }\end{array}$ & & & & \\
\hline $\begin{array}{l}\text { Empleo de estabilizantes queímicos para reducir la } \\
\text { producción de polvo }\end{array}$ & & & & \\
\hline $\begin{array}{l}\text { Utilización de toldos y lonas para la cobertura del } \\
\text { material expuesto a la intemperie, incluido su } \\
\text { transporte }\end{array}$ & & & & \\
\hline Gestión de residuos & SI & No & $C C_{6}$ & $V_{6}$ \\
\hline $\begin{array}{l}\text { Residuos especiales provenientes de los aditivos } \\
\text { utilizados }\end{array}$ & & & & \\
\hline Gestión del agua & SI & No & $\mathrm{CC}_{7}$ & $\overline{V_{7}}$ \\
\hline $\begin{array}{l}\text { Se utilizan dispositivos de ahorro de agua en los puntos } \\
\text { de consumo }\end{array}$ & & & & \\
\hline $\begin{array}{l}\text { Se utilizan contenedores para la recogida del agua de } \\
\text { lluvia y el posterior uso de la misma }\end{array}$ & & & & \\
\hline
\end{tabular}

\subsection{Definición del Índice del Proceso Constructivo}

Se define como "Índice del Proceso Constructivo" de una construcción al resultado de aplicar la siguiente expresión:

$$
\sum_{i=1}^{n} C p \cdot(1-I i / 9)
$$

dónde:

- Cp: Coeficiente de ponderación de cada impacto, tiene en cuenta la importancia relativa de cada impacto estudiado. Estos valores fueron adoptados a partir del estudio de opiniones de expertos, realizado a través de la técnica cualitativa grupo focal o "grupo de discusión" (focus group). La experiencia se realizó en una reunión de un grupo de investigadores expertos en la temática. 
Tabla 6: Coeficientes de ponderación para el IPC.

\begin{tabular}{|c|c|c|c|}
\hline \multicolumn{3}{|r|}{ IMPACTOS } & $C p$ \\
\hline \multirow{7}{*}{$\begin{array}{c}\text { Uso } \\
\text { eficiente } \\
\text { de la } \\
\text { energía }\end{array}$} & \multirow{3}{*}{$\begin{array}{l}\text { Consumo } \\
\text { de energía }\end{array}$} & Equipamiento móvil & 0,04 \\
\hline & & Máquinas / Herramientas & 0,05 \\
\hline & & Calefacción / Enfriamiento de oficinas & 0,05 \\
\hline & \multirow{2}{*}{$\begin{array}{l}\text { Iluminación } \\
\text { de la obra }\end{array}$} & Consumo de energía & 0,03 \\
\hline & & Contaminación lumínica & 0,03 \\
\hline & \multirow{2}{*}{ Transporte } & De carga & 0,05 \\
\hline & & De personal & 0,03 \\
\hline \multirow{6}{*}{$\begin{array}{l}\text { Gestión de } \\
\text { residuos }\end{array}$} & \multicolumn{2}{|c|}{ Hormigón / Acero } & 0,04 \\
\hline & \multirow{3}{*}{$\begin{array}{l}\text { Obras } \\
\text { complement } \\
\text { arias }\end{array}$} & Residuos de demolición & 0,05 \\
\hline & & Residuos de excavación & 0,03 \\
\hline & & Instalación del obrador & 0,03 \\
\hline & \multicolumn{2}{|c|}{ Residuos especiales / peligrosos } & 0,05 \\
\hline & \multicolumn{2}{|c|}{ Residuos sólidos urbanos } & 0,04 \\
\hline \multirow{4}{*}{ Agua } & \multirow{2}{*}{$\begin{array}{l}\text { Consumo } \\
\text { del agua }\end{array}$} & Consumo humano & 0,06 \\
\hline & & Consumo obras & 0,06 \\
\hline & \multirow{2}{*}{$\begin{array}{l}\text { Afectación } \\
\text { del agua }\end{array}$} & Aguas subterráneas & 0,06 \\
\hline & & Aguas superficiales & 0,06 \\
\hline \multirow{4}{*}{ Atmósfera } & \multicolumn{2}{|c|}{ Ruidos molestos } & 0,05 \\
\hline & \multicolumn{2}{|l|}{ Olores } & 0,05 \\
\hline & Calidad del & Emisiones de gases de efecto invernadero & 0,07 \\
\hline & & Material particulado & 0,07 \\
\hline
\end{tabular}

- Ii: Valor promedio de los impactos producidos sobre el medio físico (If), biótico $(I b)$ y antrópico $(I a)$. En el caso de que el impacto no aplique sobre alguno de los medios, simplemente se anulará, promediando los valores restantes.

$$
I i=\frac{I f+I b+I a}{3}
$$

\subsubsection{Determinación de los impactos}

Los impactos sobre cada medio se evaluarán sumando las puntuaciones correspondientes a las siguientes categorías (Tabla 7): 
Tabla 7: Categorías para la ponderación de los impactos.

\begin{tabular}{|c|c|c|c|c|}
\hline \multirow{2}{*}{ Categorías } & \multirow{2}{*}{ Descripción } & \multicolumn{3}{|c|}{ Puntuación ${ }^{*}$} \\
\hline & & 1 & 2 & 3 \\
\hline $\begin{array}{l}\text { Intensidad } \\
\text { (I) }\end{array}$ & $\begin{array}{lr}\text { Según } & \text { la } \\
\text { intensidad } & \text { con } \\
\text { la que } & \text { actúe } \\
\text { sobre } & \text { el } \\
\text { ambiente. } & \end{array}$ & $\mid \begin{array}{llr}\text { Bajo: } & \text { Para } & \text { un } \\
\text { impacto } & \text { cuyo } \\
\text { efecto } & \text { producirá } \\
\text { un } & \text { perjuicio } \\
\text { limitado } & \text { en } & \text { el } \\
\text { sector } & \text { afectado. }\end{array}$ & $\begin{array}{l}\text { Medio: Para un } \\
\text { impacto cuyo } \\
\text { efecto producirá } \\
\text { una destrucción o } \\
\text { desaparición del } \\
\text { factor en la zona. }\end{array}$ & $\begin{array}{l}\text { Alto: Para un } \\
\text { impacto cuyo } \\
\text { efecto implique } \\
\text { una destrucción } \\
\text { casi total del } \\
\text { factor en la } \\
\text { zona. }\end{array}$ \\
\hline $\begin{array}{l}\text { Extensión } \\
\text { (E) }\end{array}$ & $\begin{array}{l}\text { Se considera la } \\
\text { superficie } \\
\text { afectada por el } \\
\text { mismo. }\end{array}$ & $\begin{array}{l}\text { Inferior: Para un } \\
\text { impacto en una } \\
\text { superficie inferior } \\
\text { a la extensión de } \\
\text { la obra. }\end{array}$ & $\begin{array}{l}\text { Total: Para un } \\
\text { impacto en una } \\
\text { superficie similar } \\
\text { a la extensión de } \\
\text { la obra. }\end{array}$ & $\begin{array}{l}\text { Superior: Para } \\
\text { un impacto en } \\
\text { una superficie } \\
\text { superior a la } \\
\text { extensión de la } \\
\text { obra. }\end{array}$ \\
\hline $\begin{array}{l}\text { Duración } \\
\text { (D) }\end{array}$ & $\begin{array}{l}\text { Tiempo que el } \\
\text { impacto o sus } \\
\text { efectos } \\
\text { permanecen en } \\
\text { el ambiente. }\end{array}$ & $\mid \begin{array}{l}\text { Inferior: Para un } \\
\text { impacto en un } \\
\text { tiempo inferior a } \\
\text { la duración de la } \\
\text { obra. }\end{array}$ & $\begin{array}{l}\text { Total: Para un } \\
\text { impacto en un } \\
\text { tiempo similar a } \\
\text { la duración de la } \\
\text { obra. }\end{array}$ & $\begin{array}{|lrr|}\text { Superior: } & \text { Para } \\
\text { un } & \text { impacto } & \text { en } \\
\text { un } & \text { tiempo } \\
\text { mayor } & \text { a } & \text { la } \\
\text { duración } & \text { de } & \text { la } \\
\text { obra. } & & \\
\end{array}$ \\
\hline
\end{tabular}

\subsubsection{Impactos a evaluar}

En la Tabla 8 se presentan las principales categorías en las que se producen los impactos generados durante el proceso constructivo y una breve descripción de los mismos.

Tabla 8. Descripción de los Impactos a analizar.

\begin{tabular}{|c|l|}
\hline $\begin{array}{c}\text { Categorías de } \\
\text { análisis }\end{array}$ & \multicolumn{1}{|c|}{ Descripción } \\
\hline $\begin{array}{c}\text { Uso eficiente } \\
\text { de la energía }\end{array}$ & $\begin{array}{l}\text { Conjunto de acciones que permiten optimizar la relación entre la } \\
\text { cantidad de energía consumida y el producto final obtenido. Esto se } \\
\text { puede lograr a través de la implementación de diversas medidas e } \\
\text { inversiones a nivel tecnológico, de gestión y de hábitos culturales de } \\
\text { los actores sociales involucrados. (Howland, 1987), (Cepeda } \\
\text { Gutiérrez, 2008) }\end{array}$ \\
\hline $\begin{array}{c}\text { Gestión de } \\
\text { residuos }\end{array}$ & $\begin{array}{l}\text { Está relacionado con acciones normativas, operativas, financieras, } \\
\text { de planificación, administrativas, sociales, educativas, de } \\
\text { monitoreo, supervisión y evaluación, para el manejo de residuos, } \\
\text { lonsiderando desde su generación hasta la disposición final, a fin } \\
\text { de lograr: beneficios ambientales, la optimización económica de su } \\
\text { manejo y su aceptación social, respondiendo a la realidad de cada } \\
\text { lugar. (Ley 25.916) (Ley 25.831) }\end{array}$ \\
\hline
\end{tabular}




\begin{tabular}{|c|l|}
\hline Agua & $\begin{array}{l}\text { Consumo de agua utilizado en la obra, en las instalaciones } \\
\text { complementarias y dentro de las restantes obras que se desarrollen } \\
\text { en el puerto. También se pondera la afectación sobre las aguas } \\
\text { subterráneas y superficiales. (Dadon, 2009) (Ley 25.688) }\end{array}$ \\
\hline Atmósfera & $\begin{array}{l}\text { Incidencia en la emisión a la atmósfera de contaminantes que } \\
\text { pueden afectar a la salud de las personas expuestas. Entre estos } \\
\text { contaminantes se destacan: el material particulado en suspensión } \\
\text { presente en las obras y demoliciones, junto con los contaminantes } \\
\text { propios de las emisiones del tráfico de vehículos, dentro de la obra } \\
\text { y para abastecer a la misma. (Guía ambiental para terminales } \\
\text { portuarias, 2009) }\end{array}$ \\
\hline
\end{tabular}

\subsubsection{Cálculo del IPC.}

En la Tabla 9 se resume el método de obtención del Índice del Proceso Constructivo (IPC), conteniendo la información presentada en todo el ítem 3.2.

Tabla 9: Determinación del IPC.

\begin{tabular}{|c|c|c|c|c|c|c|c|c|c|c|c|c|c|}
\hline \multicolumn{14}{|c|}{ INDICE DEL PROCESO CONSTRUCTIVO } \\
\hline \multirow{2}{*}{\multicolumn{3}{|c|}{ IMPACTOS }} & & \multicolumn{3}{|c|}{ Medio físico } & \multicolumn{3}{|c|}{ Medio biótico } & \multicolumn{3}{|c|}{ M. antrópico } & \multirow{2}{*}{ TOTALES } \\
\hline & & & & 1 & D & $E$ & 1 & $\mathrm{D}$ & $\mathrm{E}$ & 1 & $\mathrm{D}$ & $E$ & \\
\hline \multirow{7}{*}{ 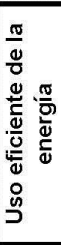 } & \multirow{3}{*}{$\begin{array}{l}\text { Consumo } \\
\text { de energía }\end{array}$} & Equipamiento móvil & 0,04 & & & & & & & & & & \\
\hline & & Máquinas / Herramientas & 0,05 & & & & & & & & & & \\
\hline & & $\begin{array}{l}\text { Calefacción / Enfriamiento } \\
\text { de oficinas }\end{array}$ & 0,05 & & & & & & & & & & \\
\hline & \multirow{2}{*}{$\begin{array}{l}\text { Iluminación } \\
\text { de la obra }\end{array}$} & Consumo de energía & 0,03 & & & & & & & & & & \\
\hline & & Contaminación lumínica & 0,03 & & & & & & & & & & \\
\hline & \multirow{2}{*}{ Transporte } & De carga & 0,05 & & & & & & & & & & \\
\hline & & De personal & 0,03 & & & & & & & & & & \\
\hline \multirow{6}{*}{ 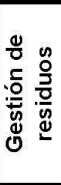 } & \multicolumn{2}{|c|}{ Hormigón / Acero } & 0,04 & & & & & & & & & & \\
\hline & \multirow{3}{*}{\begin{tabular}{|l|} 
Obras \\
complement \\
arias
\end{tabular}} & Residuos de demolición & 0,05 & & & & & & & & & & \\
\hline & & Residuos de excavación & 0,03 & & & & & & & & & & \\
\hline & & Instalación del obrador & 0,03 & & & & & & & & & & \\
\hline & \multicolumn{2}{|c|}{ Residuos especiales/peligrosos } & 0,05 & & & & & & & & & & \\
\hline & \multicolumn{2}{|c|}{ Residuos sólidos urbanos } & 0,04 & & & & & & & & & & \\
\hline \multirow{4}{*}{ 孚 } & \multirow{2}{*}{$\begin{array}{l}\text { Consumo } \\
\text { del agua }\end{array}$} & Consumo humano & 0,06 & & & & & & & & & & \\
\hline & & Consumo obras & 0,06 & & & & & & & & & & \\
\hline & \multirow{2}{*}{$\begin{array}{l}\text { Afectación } \\
\text { del agua }\end{array}$} & Aguas subterráneas & 0,06 & & & & & & & & & & \\
\hline & & Aguas superficiales & 0,06 & & & & & & & & & & \\
\hline \multirow{4}{*}{ 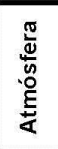 } & \multicolumn{2}{|c|}{ Ruidos molestos } & 0,05 & & & & & & & & & & \\
\hline & \multicolumn{2}{|l|}{ Olores } & 0,05 & & & & & & & & & & \\
\hline & \multirow{2}{*}{$\begin{array}{l}\text { Calidad del } \\
\text { aire }\end{array}$} & $\begin{array}{l}\text { Emisiones de gases de } \\
\text { efecto invernadero }\end{array}$ & 0,07 & & & & & & & & & & \\
\hline & & Material particulado & 0,07 & & & & & & & & & & \\
\hline
\end{tabular}




\section{Conclusiones}

Como se ha podido apreciar en este trabajo, las consideraciones ambientales relacionadas con las obras de hormigón son una importante herramienta que puede contribuir a mejorar la sustentabilidad de las mismas.

La metodología propuesta se desarrolló considerando las afectaciones ambientales generadas en la elaboración de los materiales, en su transporte, y en la construcción propiamente dicha. Para ello, se subdividió al indicador principal en dos partes. Por un lado, el IPB tienen en cuenta los impactos producidos por los materiales y productos básicos necesarios para la elaboración del hormigón, hasta su arribo a la obra, y por el otro, los correspondientes a la obra propiamente dicha, en su lugar de emplazamiento son valorados por el IPC.

Para el desarrollo de los índices se tuvo en cuenta la situación local del país, tanto en:

- Normativas de calidad;

- Tipos y distancias de transporte; y

- Tipos de impactos que más habitualmente pueden generarse en nuestro entorno.

En cuanto a los impactos durante el proceso constructivo, la metodología valora los producidos sobre los diferentes medios (físico, biótico y antrópico), a través del análisis del: uso eficiente de la energía, gestión de residuos, agua y atmósfera.

La aplicación del índice ISH permite cuantificar la sustentabilidad de una construcción de hormigón, con una metodología relativamente simple y cuya información de ingreso es de fácil acceso. 


\section{Referencias}

ALCONPAT Brasil (2013). Boletín Técnico N ${ }^{\circ}$ 7: Sostenibilidad en la construcción. Recuperado de http://alconpat.org.br/wp-content/uploads/2012/09/B7Sustentabilidade-na-Constru\%C3\%A7\%C3\%A3o.pdf (última consulta30/06/2014)

Cepeda Gutiérrez M., Mardaras Larrañaga I. (2009). Cuantificación energética de la construcción de edificios y el proceso de urbanización. Revista Conarquitectura.

Dadon J. R. (2009). Manejo Costero República Argentina, Gestión de Espacios Costeros, Facultad de Arquitectura, Diseño y Urbanismo, Universidad de Buenos Aires.

EHE (2008). Instrucción de Hormigón Estructural. Ministerio de Fomento. Gobierno de España.

Guía ambiental para terminales portuarias (2006). Ministerio de Ambiente y Desarrollo Territorial, Dirección de Desarrollo Sectorial Sostenible, Ministerio de Transporte. República de Colombia.

Howland J. J. (1987). Ahorro de recursos energéticos en la preparación, transporte y vertido de la mezcla de hormigón. Revista mensual Información a Dirigentes. Cuba.

Ley 25.688 (2002). Régimen de Gestión Ambiental de Aguas, Secretaría de Ambiente y Desarrollo Sustentable de la Nación.

Ley 25.831 (2004). Régimen de libre acceso a la Información Ambiental, Secretaría de Ambiente y Desarrollo Sustentable de la Nación.

Ley 25.916 (2004). Gestión integral de los residuos sólidos urbanos, Organismo Provincial para Desarrollo Sostenible de la Prov. de Buenos Aires.

Metha K. (2001). Concrete can be durable and environmentally friendly. Concrete International, Estados Unidos.

Montero M. C., Rumi L., Ortega N. F. (2013). Sustentabilidad en construcciones portuarias: Indicadores para el análisis de las obras de Hormigón Armado. 4to Congreso de Ciencias Ambientales - COPIME 2013, Buenos Aires.

Naik T. R., Moriconi G. (2005). Environmental-friendly durable concrete made with recycled materials for sustainable concrete construction. UWM Center for ByProducts Utilization, University of Wisconsin-Milwaukee, Milwaukee, USA.

Norma ISO 14001 (2004). Sistemas de Gestión Ambiental Requisitos con orientación a su uso. International Organization for Standarization (ISO).

Schneider M., Romer M., Tschudin M., Bolio H. (2011). Sustainable cement production - present and future. Cement and Concrete Research 41. 